agreement with the $\mathrm{X}$-ray results. In fact if we leave out Harrington's value for the viscosity (which seems certainly to be too low), and use the mean of the remaining values, Millikan's measurements give $e=4 \cdot 806 \times 10^{-10}$ E.s.U. If we also omit Bond's and Kellström's values, which seem likely to be too highthat is, if we only use the most recent three measurements of the viscosity-Millikan's value becomes $4.803 \times 10^{-10}$-in exact agreement with the X-ray measurements.

Queen Mary College,

(University of London), E.1.

H. R. RoBInson. June 11.

${ }^{1}$ Banerjea and Pattanaik, Nature, 141, 1016 (June 4, 1938).

2 Bäcklin and Flemberg, NATURE, 137, 655 (1936).

${ }^{3}$ Ishida, Fukushima and Suetsugu, Sci. Pap. Inst. Phys. Chem. Res., Tokyo, 709, 57 (1937).

' Harrington, Phys. Rev., 8, 738 (1916).

${ }^{5}$ Bond, Nature, 137, 1031 (1936) ; Proc. Phys. Soc., 49, 205 (1937).

- Kellström, Nature, 136, 682 (1935); Phys. Rev., 50, 190 (1936).

'Houston, Phys. Rev., 52, 751 (1937).

${ }^{8}$ Rigden, NATURe, 141, 82 (1938); Phil. 'Mag., 25, 961 (June 1938).

\section{Waves Associated with Moving Corpuscles}

By appropriate general treatment, it may be shown that solutions involving the path time are invariant, that is, can exist in any set of space co-ordinates. Starting, then, with the solution for the electron velocity ripple in terms of the transit half-angle ${ }^{1}$, it may be shown, by analogy with van der Pol's treatment of waves in $n$ dimensions ${ }^{2}$, that this ripple satisfies certain conditions. The most important of these is that the ripple satisfies a five-dimensional wave equation, the wave velocity being that of the particle, provided the frequency of the ripple is such that $\theta \gg \sqrt{ } 2$, where $\theta$ is the transit half angle $(=\omega \tau / 2)$. It will be convenient to regard this ripple as that part of the particle vibration which corresponds to free, as distinct from forced, oscillation. The factor $\frac{1}{2}$ must be associated with the fifth dimension, as follows :

$$
\theta=\omega \tau / 2=\omega\left(t-t_{1}\right) / 2=\omega r_{5} / c_{5}
$$

where $c_{5}$ is the velocity appropriate to the waves.

I have confirmed that, when no 'weight factor' is involved, the result of integrating transit time solutions over all possible values of $\theta$ (that is, from $\theta=0$ to $\theta=\infty$ ) leaves the four-dimensional solution that would have been obtained had the transit time been taken as negligible. Thus $\left(i_{c}=\right.$ electron convection current, $\bar{i}=$ total current density (ripples).

$\left[i_{c}\right]_{4}=\bar{i} e^{i \omega t} \int_{\theta=0}^{\theta=\infty} \Upsilon_{3}(2 i \theta) d i \theta=\bar{\imath} e^{i \omega t}\left|\frac{e^{-2 i \theta}-1}{2 i \theta}\right|_{0}^{\infty}=\bar{i} e^{i \omega t}$

9 Cranley Road,

W. E. Benham.

Ilford, Essex.

${ }^{1}$ Benham, W. E., Phil Mag., 5, 648 (March 1928).

${ }^{2}$ van der Pol, B., Physica, 3, 385-392 (June 1936).

\section{A Useful Statistical Test}

THE method described below gives a useful criterion of the significance of the difference between an observed and a hypothetical frequency distribution. Since it provides an exact test, it is particularly useful in the case of small samples, in which the ex. pectation in some frequency classes may be very small. It is only applicable when the number of possible samples containing the same number of observations as the observed sample is determinable. The test answers the question: Is this a reasonably likely sample to be obtained in a random draw?
It does not give the proportion of less likely samples which may be expected to arise, as does the $\chi^{2}$-test.

Let us suppose that the hypothetical population shows the following proportional frequencies in four classes :

the total being 8 .

$$
\frac{1}{8}, \frac{3}{8}, \frac{3}{8}, \frac{1}{8},
$$

The number $(N)$ of possible samples of $n$ with $r$ frequency classes is given by

$$
{ }_{r} H_{n} \text { or } \frac{(r+n-1) !}{(r-1) ! n !}
$$

Thus the number of possible samples of 8 in 4 classes is

$$
\frac{11 !}{3 ! 8 !} \text { or } 165
$$

Clearly the average probability of the samples is $1 / N$ or $1 / 165$. An observed sample having average probability cannot, of course, be considered as an unlikely sample to arise. Half the average probability has been found to separate samples at about the 5 per cent point, agreeing closely with $\chi^{2}(p=0.05)$ in cases where both tests are applicable. It is only necessary, therefore, to express $p$, the probability of an observed sample, as a fraction of $1 / N$, considering the sample as unlikely to have arisen from the hypothetical population if $N p$ is less than $0 \cdot 5$.

For example, is a sample of eight having the frequencies $3,1,1,3$, likely to have arisen as a random sample from the population specified above? $N=165$, and $p$ is given as a term in the multinomial distribution. Thus,

$$
N p=165 \times \frac{8 !}{3 ! 1 ! 1 ! 3 !} \quad\left(\frac{1}{8}\right)^{3}\left(\frac{3}{8}\right)^{1}\left(\frac{3}{8}\right)^{1}\left(\frac{1}{8}\right)^{3}=<0 \cdot 1
$$

The sample is, therefore, very unlikely to have arisen as a random sample from the hypothetical population.

The change in the number of possible samples with a change in $r$ corresponds to a change in the number of degrees of freedom with the $\chi^{2}$-test. Other restrictions on variation, such as the use of a calculated mean or standard deviation, do not change the number of possible samples at all in the same way. This suggests that restriction in the $\chi^{2}$-distribution is much more accurately allowed for by degrees of freedom in the case of differences in the numbers of frequency classes, than it is with restrictions imposed by the use of calculated statistics in specification of hypothetical populations.

Fisheries Laboratory,

H. J. Buchanan-Wollaston.

Lowestoft.

June 22.

\section{Passivity of Iron to Natural Waters}

THE passivity of iron has been known for more than a century, but until recently interest has mainly been directed to treatments which render iron inert to copper salts; such treatments do not necessarily prevent rusting by natural waters. Three sets of observations in this laboratory have indicated how passivity to such waters might be obtained. (1) The film present on iron rendered 'passive' in chromate appears to be the air-formed film with its weakest places fortified with chromium oxide ${ }^{1}$; a 'patchwork' is not ideal for protection. (2) The coarser the grinding of an iron surface, the longer the time needed to produce passivity ${ }^{2}$. (3) Susceptibility to corrosion, as judged by statistical studies, varies from spot to spot and is influenced by chemical character ${ }^{3}$. 